\title{
Fuchs Endothelial Corneal Dystrophy: Strong Association with rs613872 Not Paralleled by Changes in Corneal Endothelial TCF4 mRNA Level
}

\author{
Monika Ołdak, ${ }^{1,2}$ Ewelina Ruszkowska, ${ }^{1,2,3}$ Monika Udziela, ${ }^{4}$ Dominika Oziębło, \\ Ewelina Bińczyk, ${ }^{4}$ Aneta Ścieżyńska, ${ }^{2}$ Rafal Płoski, ${ }^{5}$ and Jacek P. Szaflik ${ }^{4}$ \\ ${ }^{1}$ Department of Genetics, World Hearing Center, Institute of Physiology and Pathology of Hearing, Warsaw, Poland \\ ${ }^{2}$ Department of Histology and Embryology, Medical University of Warsaw, Warsaw, Poland \\ ${ }^{3}$ Postgraduate School of Molecular Medicine, Medical University of Warsaw, Warsaw, Poland \\ ${ }^{4}$ Department of Ophthalmology, Medical University of Warsaw, Warsaw, Poland \\ ${ }^{5}$ Department of Medical Genetics, Medical University of Warsaw, Warsaw, Poland \\ Correspondence should be addressed to Monika Ołdak; m.oldak@ifps.org.pl
}

Received 27 March 2015; Revised 5 June 2015; Accepted 8 June 2015

Academic Editor: Alessandro Lambiase

Copyright (C) 2015 Monika Ołdak et al. This is an open access article distributed under the Creative Commons Attribution License, which permits unrestricted use, distribution, and reproduction in any medium, provided the original work is properly cited.

\begin{abstract}
Fuchs endothelial corneal dystrophy (FECD) is a common corneal endotheliopathy with a complex and heterogeneous genetic background. Different variants in the TCF4 gene have been strongly associated with the development of FECD. TCF4 encodes the E2-2 transcription factor but the link between the strong susceptibility locus and disease mechanism remains elusive. Here, we confirm a strong positive association between TCF4 single nucleotide polymorphism rs613872 and FECD in Polish patients (OR $=12.95,95 \%$ CI: 8.63-19.42, $\left.\chi^{2}=189.5, p<0.0001\right)$. We show that TCF4 expression at the mRNA level in corneal endothelium $(n=63)$ does not differ significantly between individuals with a particular TCF4 genotype. It is also not altered in FECD patients as compared to control samples. The data suggest that changes in the transcript level containing constitutive TCF4 exon encoding the amino-terminal part of the protein seem not to contribute to disease pathogenesis. However, considering the strong association of TCF4 allelic variants with FECD, genotyping of TCF4 risk alleles may be important in the clinical practice.
\end{abstract}

\section{Introduction}

Fuchs endothelial corneal dystrophy (FECD) affects approximately $4 \%$ of the population over the age of 40 and is the most common genetic disorder of the corneal endothelium. The disease usually has a late onset and presents clinically during the fifth or sixth decade of life [1]. It is characterized by thickening of Descemet's membrane and deposition of extracellular matrix in the form of guttae [2]. Patients with FECD have a reduced density of corneal endothelial cells. As the cells regulate corneal hydration and maintain its transparency, their loss may eventually progress to corneal oedema and vision loss [1]. For the end-stage disease transplant surgery represents the only definitive treatment. FECD is a leading indication for corneal transplantation and Descemet's
Stripping Automated Endothelial Keratoplasty (DSAEK) is considered a standard procedure for these patients [3].

Genetic basis for FECD is complex and heterogeneous. Early-onset form of endothelial dystrophy with some of the phenotypic features of FECD occurs rarely and displays an autosomal dominant mode of inheritance with mutations in the COL8A2 gene (1p34.3, MIM*120252). The more common late-onset FECD begins usually after the age of 40 years and both familial and sporadic FECD cases have been described. Familial late-onset FECD is inherited in autosomal dominant fashion. However, the majority of late-onset FECD cases are sporadic with a negative family history. It is much more common and severe in females than in males (3$4: 1$ ] [4], which contradicts a strictly autosomal dominant transmission but follows multifactorial inheritance. Higher 
TABLE 1: Loci, genes, and genetic variants related to late-onset FECD.

\begin{tabular}{lcc}
\hline Chromosome & Locus/gene/variant & Late-onset FECD \\
\hline $1 \mathrm{p} 35.1$ & $\mathrm{rs} 760594$ & $\mathrm{~F}$ \\
$5 \mathrm{q} 12.3$ & $\mathrm{rs} 1301475$ & $\mathrm{~F}$ \\
$5 \mathrm{q} 33.1-\mathrm{q} 35.2$ & $\mathrm{FCD} 3$ & $\mathrm{~F}$ \\
$7 \mathrm{q} 22.3$ & $\mathrm{rs} 257376$ & $\mathrm{~F}$ \\
$8 \mathrm{p} 21.3$ & $\mathrm{rs} 2466216$ & $\mathrm{~F}$ \\
$8 \mathrm{p} 21.1$ & $\mathrm{rs} 9797$ & $\mathrm{~F}$ \\
$8 \mathrm{q} 21.13$ & $\mathrm{rs} 1380229$ & $\mathrm{~F}$ \\
9p22.1-p24.1 & $\mathrm{FCD} 4$ & $\mathrm{~F}$ \\
$10 \mathrm{p} 11.22$ & ZEB1 & $\mathrm{F} / \mathrm{S}$ \\
$10 \mathrm{q} 24.2$ & $\mathrm{rs} 1889974$ & $\mathrm{~F}$ \\
$13 \mathrm{pter}-\mathrm{q} 12.13$ & $\mathrm{FCD} 1$ & $\mathrm{~F}$ \\
$15 \mathrm{q} 22.2$ & $\mathrm{rs} 235512$ & $\mathrm{~F}$ \\
$15 \mathrm{q} 22.31$ & $\mathrm{rs} 352476$ & $\mathrm{~F}$ \\
$15 \mathrm{q} 25.3$ & AGBL1 & $\mathrm{F} / \mathrm{S}$ \\
$17 \mathrm{q} 25.3$ & $\mathrm{rs} 938350$ & $\mathrm{~F}$ \\
$18 \mathrm{q} 21.1$ & $L O X H D 1$ & $\mathrm{~F} / \mathrm{S}$ \\
$18 \mathrm{q} 21.2$ & TCF4 & $\mathrm{F} / \mathrm{S}$ \\
$18 \mathrm{q} 21.2-\mathrm{q} 21.32$ & $\mathrm{FCD} 2$ & $\mathrm{~F}$ \\
$20 \mathrm{p} 13$ & SLC4A11 & $\mathrm{F} / \mathrm{S}$ \\
$20 \mathrm{p} 12.2$ & $\mathrm{rs674630}$ & $\mathrm{F}$ \\
Xq28 & $\mathrm{rs} 1990383$ & $\mathrm{~F}$ \\
\hline
\end{tabular}

F: familial, S: sporadic.

risk of cornea guttata was independently associated with older age, female sex, and a thinner central corneal thickness [5] as well as genetic variants [6].

To date, four different genes ZEB1 (10p11.22, MIM*189909), AGBL1 (15q25.3, MIM*615496), LOXHD1 (18q21.1, MIM*613072), and SLC4A11 (20p13, MIM*610206) as well as four causal loci on chromosomes 5q33.1-q35.2 (FCD3), 9p22.1-p24.1 (FCD4), 13pter-q12.13 (FCD1), and $18 \mathrm{q} 21.2-\mathrm{q} 21.32$ (FCD2), together with a number of susceptibility loci, have been implicated in the pathogenesis of FECD (summarized in Table 1) [7, 8]. A strong association has been established between TCF4 gene (18q21.2, MIM*602272) variants and FECD. A genome-wide association study has identified a common biallelic deep intronic TCF4 single nucleotide polymorphism (SNP, rs613872; NG_011716.1:g.50559C>A) as a highly significant risk factor for FECD [6]. In addition to that, a separate study reported a trinucleotide expansion (CTG18.1) in the intron region of TCF4, which is highly prevalent in FECD individuals [9]. The role of these associations in FECD development remains poorly understood. In the study we set out to analyze whether the presence of TCF4 rs613872 risk allele affects the expression of TCF4 in corneal endothelial cells, which would provide a functional link between a risk factor and disease mechanism in FECD.

\section{Patients and Methods}

The study conformed to the tenets of the Declaration of Helsinki and was approved by the local Ethics Committee.
Patients were recruited from the Department of Ophthalmology, Medical University of Warsaw, and gave informed consent prior to participation. The diagnosis of FECD was based on the visualization of "guttae" and stromal oedema by slit-lamp examination, confocal microscopy in vivo (IVCM, Confoscan 4, Nidek Technologies), and anterior segment optical coherence tomography (CASIA Cornea/Anterior Segment OCT SS-1000, Tomey) (Figure 1).

2.1. Genotyping. Genomic DNA was isolated from peripheral blood samples of sporadic, unrelated FECD patients $(n=$ 252; 187 females and 65 males) with a standard salting-out procedure. Control DNA samples $(n=323)$, representative of the background population of central Poland, came from the repository of the Department of Medical Genetics [10]. The TCF4 rs613872 was genotyped using ABI Custom TaqMan SNP Genotyping Assay (Applied Biosystems) and the realtime PCR system (Viia7, Thermo Fisher Scientific). The accuracy of genotyping was confirmed by Sanger sequencing in selected subjects.

2.2. RNA Isolation and Quantitative Real-Time PCR. Corneal endothelial cell monolayers attached to Descemet's membrane were obtained from FECD patients $(n=40)$ during endothelial keratoplasty (DSAEK) or excised from donor corneoscleral buttons $(n=23)$ that were not used for transplantation. The FECD patients and controls included in the RNA studies derived from the group genotyped at rs613872 (FECD group: TT $n=3$, TG $n=31$, GG $n=6$; control group: TT $n=14$, TG $n=6$, GG $n=3$ ). The specimens were submerged in the RNAlater storage solution (Ambion, Austin, TX, USA) and frozen immediately. Total RNA was extracted using Trizol (Thermo Fisher Scientific, Waltham, MA, USA) and cDNA was generated from $500 \mathrm{ng}$ of RNA with the Maxima First Strand cDNA Synthesis Kit (Thermo Fisher Scientific). Gene expression was measured by real-time PCR (Viia7, Thermo Fisher Scientific) using PCR primers (Oligo, Warsaw, Poland) and probes (Roche Universal Probe Library) designed with the ProbeFinder software, version 2.50 (Roche). The analyzed coding region of TCF4 is present in both TCF4-A and TCF4-B isoforms. It is located in the amino-terminal part of the protein, close to the activation domain 2 (AD2). For TCF4 quantitative PCR (qPCR), the primer pair $5^{\prime}$-GCACTTTCCCTAGCTCCTTCT $-3^{\prime}$ and $5^{\prime}$-GCATAGCCAGGCTGATTCAT $-3^{\prime}$ and probe 25 , for RPL13A (60S ribosomal protein L13a) qPCR the primer pair $5^{\prime}$-CTGGTGCTTGATGGTCGAG- $3^{\prime}$ and $5^{\prime}$-GTTGATGCCTTCACAGCGTA-3' and probe 77 were used. Expression values for TCF4 were calculated using the modified double delta $\mathrm{Ct}(\Delta \Delta \mathrm{Ct})$ method and absolute quantification and normalized to RPL13A [11].

2.3. Statistical Analysis. Hardy-Weinberg equilibrium (HWE) was analyzed with the $\chi^{2}$ test in the patient and control groups. The odds ratios (ORs) with 95\% confidence intervals $(95 \% \mathrm{CI})$ and $p$ values were calculated with the Web-Assotest program (http://www.ekstroem.com/assotest/ assotest.html). Differences in gene expression between 


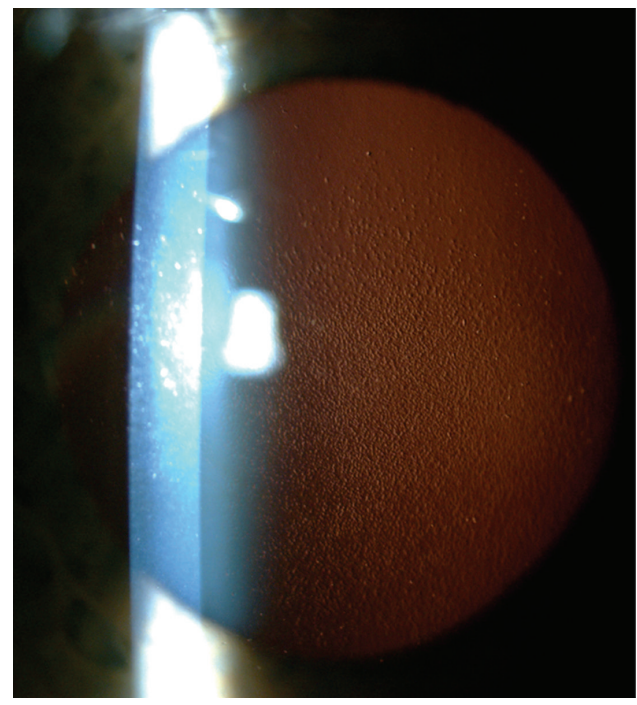

(a)

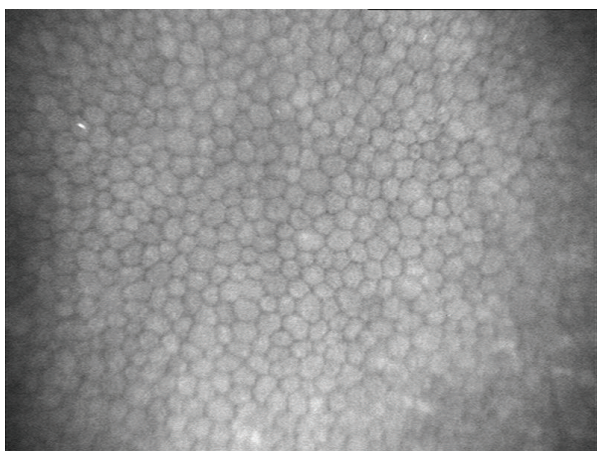

(b)

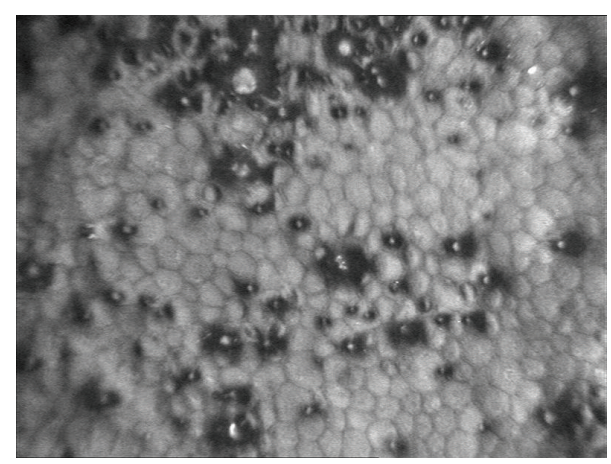

(c)

Figure 1: Characteristic features of FECD. (a) Slit-lamp photography shows the presence of pathological guttae, focal excrescences of Descemet's membrane at the level of corneal endothelium in a patient with FECD. (b) Confocal microscopy image of the corneal endothelium in a control subject demonstrates a regular mosaic of the endothelial monolayer with bright cell bodies and dark, hexagonal cell boundaries. (c) Confocal microscopy in a patient with FECD reveals pleomorphism and polymegathism of the endothelium and typical guttae as dark bodies with a central bright reflex.

TABLE 2: Genotype distribution and allele frequency of TCF4 rs613872 in patients with FECD and control subjects.

\begin{tabular}{|c|c|c|c|c|c|c|c|c|}
\hline \multirow{2}{*}{$\begin{array}{l}\text { TCF4 rs613872 } \\
\text { genotype }\end{array}$} & \multicolumn{4}{|c|}{ Genotype counts, $n(\%)$} & \multirow{2}{*}{$\begin{array}{l}\text { OR dominant model } \\
\qquad(95 \% \mathrm{CI})\end{array}$} & \multicolumn{2}{|c|}{ Allele counts, $n(\%)$} & \multirow{2}{*}{$\begin{array}{c}\chi^{2} \text { statistic, } \\
p\end{array}$} \\
\hline & TT & TG & GG & Total & & $\mathrm{T}$ & G & \\
\hline FECD patients & $46(18.25 \%)$ & $170(67.46 \%)$ & $36(14.29 \%)$ & 252 & & $262(51.98 \%)$ & $242(48.02 \%)$ & 156.7 \\
\hline Controls & $240(74.30 \%)$ & $74(22.91 \%)$ & $9(2.79 \%)$ & 323 & $(8.63-19.42)$ & $554(85.76 \%)$ & $92(14.24 \%)$ & $<0.0001$ \\
\hline
\end{tabular}

the groups were analyzed with a two-sided unpaired $t$-test (Statistica, StatSoft, Poland). Pearson's correlation was used to assess the degree of the relationship between gene expression and age or sex. $p<0.05$ was considered to indicate statistical significance.

\section{Results}

Both the patient and control groups were genotyped for the TCF4 SNP rs613872. In FECD patients the distribution of the TT, TG, and GG genotypes showed a significant deviation from Hardy-Weinberg equilibrium $\left(\chi^{2}=31.1, p<\right.$ $0.0001)$, while in control subjects the genotype distribution remained in the Hardy-Weinberg equilibrium $\left(\chi^{2}=1.3\right.$, $p=0.265)$. Allele $\mathrm{G}$ was significantly more prevalent in patients with FECD than among control subjects (Table 2). The odds ratio (OR) for two copies of the risk allele (GG homozygotes versus TT homozygotes) was 20.87 (95\% CI: 9.42-46.24), whereas the OR for one copy of the risk allele G (TG heterozygotes versus TT homozygotes) was 11.99 (95\% CI: 7.90-18.19). Testing the association between FECD and the TCF4 genotype under dominant, additive, or recessive models, we found that the most plausible model was the 


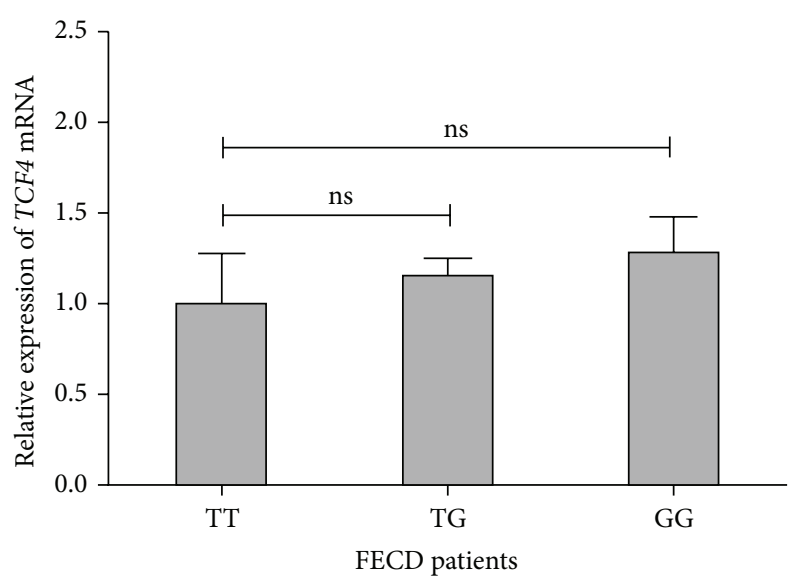

(a)

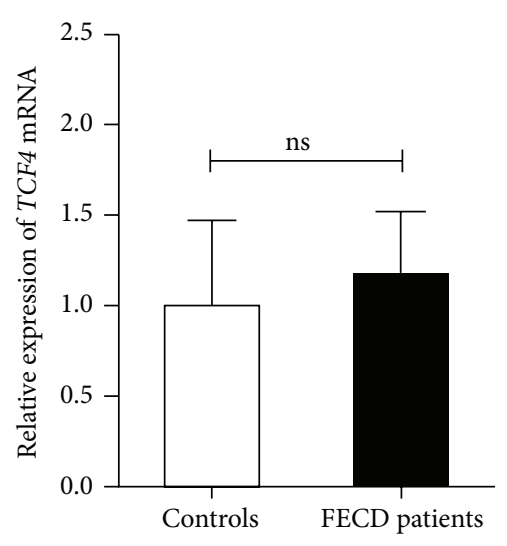

(b)

FIGURE 2: Expression of TCF4 in corneal endothelial cells. The amount of TCF4 mRNA was quantified by real-time PCR in relation to RPL13A. (a) Expression of TCF4 in FECD patients with respect to a different TCF4 genotype at $\operatorname{rs613872}$ (TT $n=3$, TG $n=31$, and GG $n=6$ ) is shown; ns: nonsignificant. (b) Average values of TCF4 mRNA expression in control samples (white bars) and FECD patients (black bars) are shown; ns: nonsignificant.

dominant one whereas the additive and recessive models could be formally rejected as indicated by the $p$ values for model fit $(p<0.0001)$. The dominant model (combining the GG and TG into one category) conferred the highest OR of all the tested models (OR $=12.95,95 \%$ CI: 8.63-19.42, $\left.\chi^{2}=189.5, p<0.0001\right)$.

Expression of TCF4 was not age or sex dependent. There were no differences in TCF4 mRNA levels in FECD patients with a particular TCF4 SNP rs613872 genotype (Figure 2(a)) but also when TCF4 genotypes of patients and controls were analyzed together (data not shown). No increase in the amount of TCF4 mRNA transcripts was observed in FECD patients as compared to the control group (Figure 2(b)).

\section{Discussion}

Our data are in accord with previous studies showing that the presence of a TCF4 risk allele at rs613872 is much more common in Caucasian patients with FECD and strongly predisposes to the development of the corneal dystrophy $[6,12-16]$. Indian FECD patients do not show a distinct association with this TCF4 variant, which may be explained by a small sample size studied [17]. In Chinese FECD patients no association could be observed as the population is not polymorphic at this genomic position [18-20]. In these FECD patients, TCF4 genetic variants adjacent to rs613872 (e.g., rs17089887 in both Indian and Chinese subjects) were strongly associated with FECD, which suggested the presence of significant disease-causing changes in the nearby regions of these alleles $[17,19,20]$. All the relevant SNPs in the respective populations are in strong linkage disequilibrium with a newly identified CTG18.1 trinucleotide repeat expansion in the TCF4 gene $[9,17,20,21]$, which confers a transethnic association with FECD.

TCF4 is now widely recognized as a major contributor to FECD. One may assume that patients carrying TCF4 risks alleles and thus having an increased susceptibility to FECD should more often have follow-up ophthalmic examinations. Diagnosis of cataract in these patients might be an indication for an earlier cataract surgery before a full-blown, clinically significant FECD develops and the patients require a combined procedure of corneal transplantation and cataract surgery.

TCF4 genetic variants are strongly associated with FECD but so far little is known about a possible involvement of TCF4 gene products in the development of FECD. Hypothesizing that the strong genetic association may indicate a causal relationship and the TCF4 genetic variant may represent a tissuespecific regulatory element, TCF4 mRNA studies in corneal endothelial cells were performed. Analyzing more than 60 different corneal endothelium and Descemet's membrane complexes, we have not found any significant differences in the expression of TCF4 at mRNA level in FECD patients as compared to control samples. There were no differences in TCF4 expression between the carriers and noncarriers of the TCF4 risk allele neither in the group of FECD patients alone nor when FECD patients and controls were pooled together. The data suggest that changes in the transcript level containing constitutive TCF4 exon encoding the aminoterminal part of the protein seem not to contribute to disease pathogenesis.

Recently, unaltered TCF4 expression was also reported in another group of FECD patients [22]. In the absence of distinguished differences in the amount of mRNA, transcript activity still may be altered or posttranslational mechanisms may affect protein structure, level, or cellular distribution. A novel and so far the only identified link between TCF4 susceptibility and FECD disease mechanism is the formation of toxic poly(CUG)n RNA and missplicing events in patients with TCF4 repeat expansion. In corneal endothelial cells of these patients the TCF4 repeats are actively transcribed and seem to preferentially accumulate into poly(CUG) RNA foci. 
A protein found to be immobilized in RNA foci is MBNL1, a splicing regulator. Consequently, differential splicing events were found in corneal endothelium of FECD patients [23].

Our study confirms and extends the association of TCF4 with FECD by testing a novel previously not analyzed population. It advances our knowledge on the role of TCF4 in the development of FECD. Genotyping of TCF4 risk alleles might be considered as a diagnostic procedure as the genetic results have an important predictive value and are of clinical utility.

\section{Conflict of Interests}

The authors declare no conflict of interests regarding this paper.

\section{Acknowledgments}

This work was supported by the Medical University of Warsaw Grants 1M15/NM4/2011, 1M15/N/2015, and 2WF/N/ 2015 and the project entitled "Integrated System of Tools Designed for Diagnostics and Telerehabilitation of the Sense Organs Disorders (Hearing, Vision, Speech, Balance, Taste, Smell)" INNOSENSE, cofinanced by the National Centre for Research and Development within the STARTEGMED Program.

\section{References}

[1] J. Zhang and D. V. Patel, "The pathophysiology of Fuchs' endothelial dystrophy-a review of molecular and cellular insights," Experimental Eye Research, vol. 130, pp. 97-105, 2015.

[2] S. G. Levy, J. Moss, H. Sawada, P. J. C. Dopping-Hepenstal, and A. C. E. McCartney, "The composition of wide-spaced collagen in normal and diseased Descemet's membrane," Current Eye Research, vol. 15, no. 1, pp. 45-52, 1996.

[3] M. A. Nanavaty, X. Wang, and A. J. Shortt, "Endothelial keratoplasty versus penetrating keratoplasty for Fuchs endothelial dystrophy," Cochrane Database of Systematic Reviews, no. 7, Article ID CD008420, 2011.

[4] G. K. Klintworth, "Corneal dystrophies," Orphanet Journal of Rare Diseases, vol. 4, no. 1, article 7, 2009.

[5] A. Higa, H. Sakai, S. Sawaguchi et al., "Prevalence of and risk factors for cornea guttata in a population-based study in a southwestern island of Japan: the Kumejima study," Archives of Ophthalmology, vol. 129, no. 3, pp. 332-336, 2011.

[6] K. H. Baratz, N. Tosakulwong, E. Ryu et al., "E2-2 protein and Fuchs's corneal dystrophy," The New England Journal of Medicine, vol. 363, no. 11, pp. 1016-1024, 2010.

[7] B. W. Iliff, S. A. Riazuddin, and J. D. Gottsch, "The genetics of Fuchs' corneal dystrophy," Expert Review of Ophthalmology, vol. 7, no. 4, pp. 363-375, 2012.

[8] S. A. Riazuddin, S. Vasanth, N. Katsanis, and J. D. Gottsch, "Mutations in AGBL1 cause dominant late-onset Fuchs corneal dystrophy and alter protein-protein interaction with TCF4," The American Journal of Human Genetics, vol. 93, no. 4, pp. 758-764, 2013.
[9] E. D. Wieben, R. A. Aleff, N. Tosakulwong et al., "A common trinucleotide repeat expansion within the transcription factor 4 (TCF4, E2-2) gene predicts Fuchs corneal dystrophy," PLoS ONE, vol. 7, no. 11, Article ID e49083, 2012.

[10] J. P. Szaflik, M. Ołdak, R. B. Maksym et al., "Genetics of Meesmann corneal dystrophy: a novel mutation in the keratin 3 gene in an asymptomatic family suggests genotype-phenotype correlation," Molecular Vision, vol. 14, pp. 1713-1718, 2008.

[11] M. W. Pfaffl, "A new mathematical model for relative quantification in real-time RT-PCR," Nucleic Acids Research, vol. 29, no. 9, article e45, 2001.

[12] S. A. Riazuddin, E. J. McGlumphy, W. S. Yeo, J. Wang, N. Katsanis, and J. D. Gottsch, "Replication of the TCF4 intronic variant in late-onset Fuchs corneal dystrophy and evidence of independence from the FCD2 locus," Investigative Ophthalmology and Visual Science, vol. 52, no. 5, pp. 2825-2829, 2011.

[13] J. F. Stamler, B. R. Roos, M. D. Wagoner et al., "Confirmation of the association between the TCF4 risk allele and Fuchs endothelial corneal dystrophy in patients from the Midwestern United States," Ophthalmic Genetics, vol. 34, no. 1-2, pp. 32-34, 2013.

[14] Y.-J. Li, M. A. Minear, J. Rimmler et al., "Replication of tcf4 through association and linkage studies in late-onset fuchs endothelial corneal dystrophy," PLoS ONE, vol. 6, no. 4, Article ID e18044, 2011.

[15] R. P. Igo Jr., L. J. Kopplin, P. Joseph et al., "Differing roles for TCF4 and COL8A2 in central corneal thickness and fuchs endothelial corneal dystrophy," PLoS ONE, vol. 7, no. 10, Article ID e46742, 2012.

[16] A. Kuot, A. W. Hewitt, K. Griggs et al., "Association of TCF4 and CLU polymorphisms with Fuchs' endothelial dystrophy and implication of CLU and TGFBI proteins in the disease process," European Journal of Human Genetics, vol. 20, no. 6, pp. 632-638, 2012.

[17] G. G. Nanda, B. Padhy, S. Samal, S. Das, and D. P. Alone, "Genetic association of TCF4 intronic polymorphisms, CTG18.1 and rs17089887, with Fuchs' endothelial corneal dystrophy in an Indian population," Investigative Ophthalmology \& Visual Science, vol. 55, no. 11, pp. 7674-7680, 2014.

[18] K. J. Wang, V. Jhanji, J. Chen et al., "Association of transcription factor 4 (TCF4) and protein tyrosine phosphatase, receptor type G (PTPRG) with corneal dystrophies in southern Chinese," Ophthalmic Genetics, vol. 35, no. 3, pp. 138-141, 2014.

[19] A. Thalamuthu, C. C. Khor, D. Venkataraman et al., "Association of TCF4 gene polymorphisms with Fuchs' corneal dystrophy in the Chinese," Investigative Ophthalmology \& Visual Science, vol. 52, no. 8, pp. 5573-5578, 2011.

[20] C. Xing, X. Gong, I. Hussain et al., "Transethnic replication of association of CTG18.1 repeat expansion of TCF4 gene with Fuchs' corneal dystrophy in Chinese implies common causal variant," Investigative Ophthalmology \& Visual Science, vol. 55, no. 11, pp. 7073-7078, 2014.

[21] V. V. Mootha, X. Gong, H.-C. Ku, and C. Xing, "Association and familial segregation of CTG18.1 trinucleotide repeat expansion of TCF4 gene in Fuchs' endothelial corneal dystrophy," Investigative Ophthalmology and Visual Science, vol. 55, no. 1, pp. 3342, 2014.

[22] V. V. Mootha, I. Hussain, K. Cunnusamy et al., "TCF4 triplet repeat expansion and nuclear RNA foci in Fuchs' endothelial 
corneal dystrophy," Investigative Opthalmology \& Visual Science, vol. 56, no. 3, pp. 2003-2011, 2015.

[23] J. Du, R. A. Aleff, E. Soragni et al., "RNA toxicity and missplicing in the common eye disease fuchs endothelial corneal dystrophy," The Journal of Biological Chemistry, vol. 290, no. 10, pp. 5979-5990, 2015. 


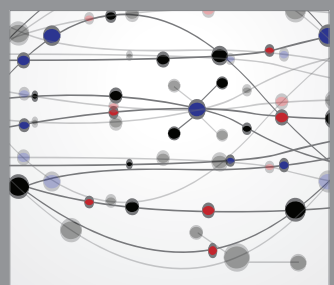

The Scientific World Journal
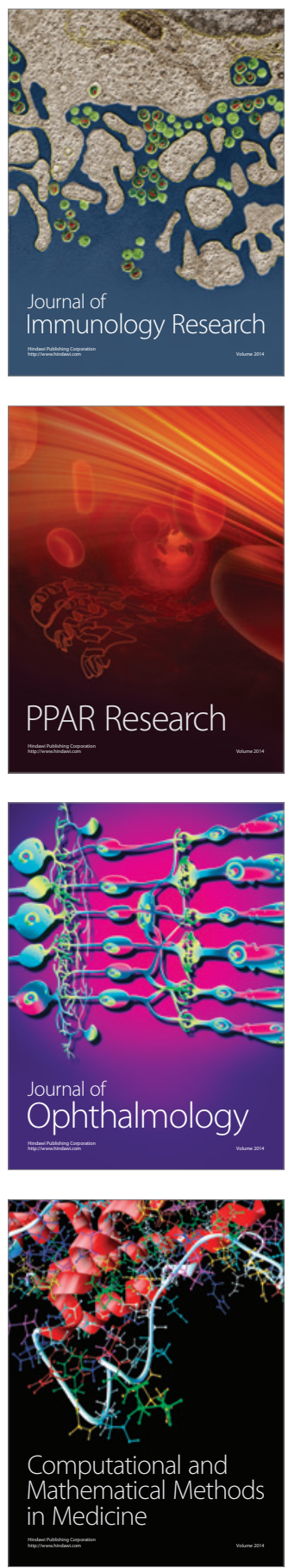

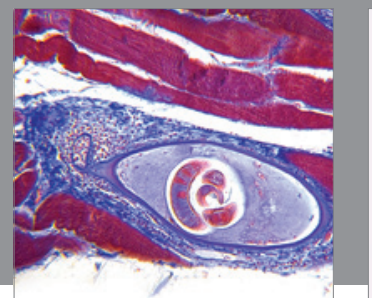

Gastroenterology

Research and Practice
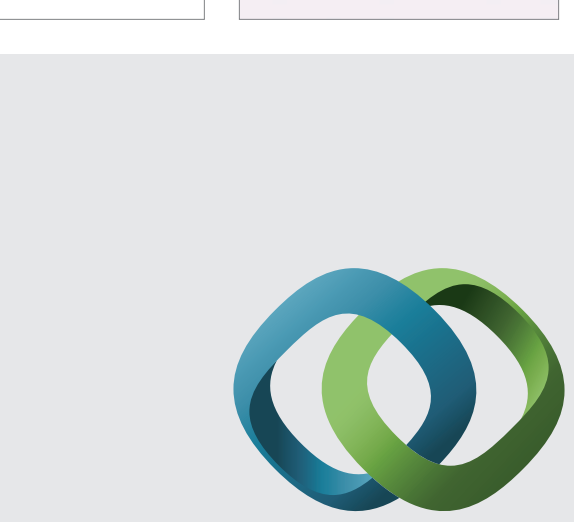

\section{Hindawi}

Submit your manuscripts at

http://www.hindawi.com
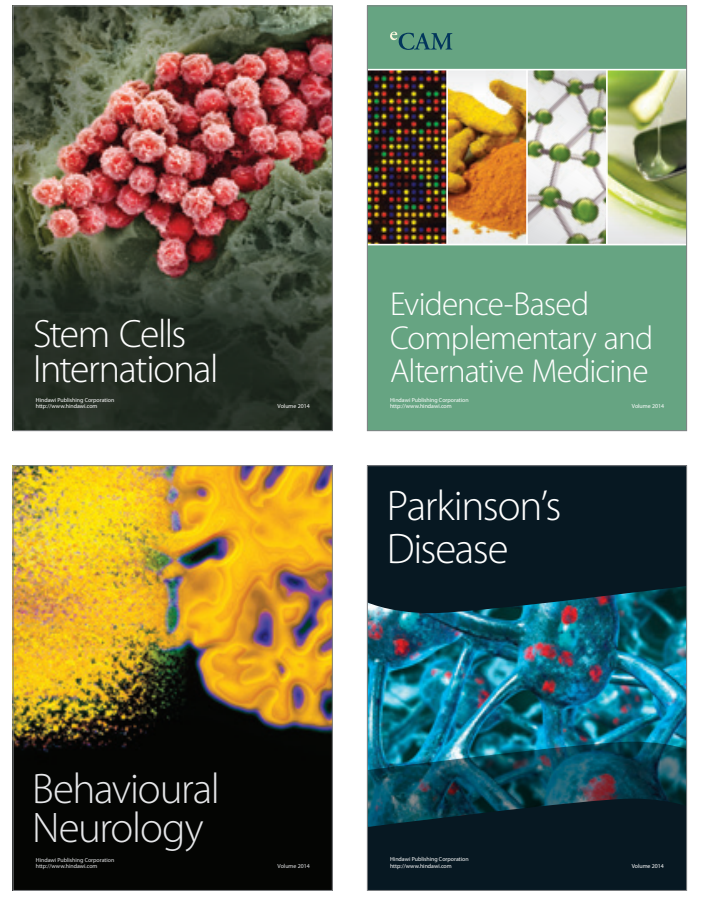
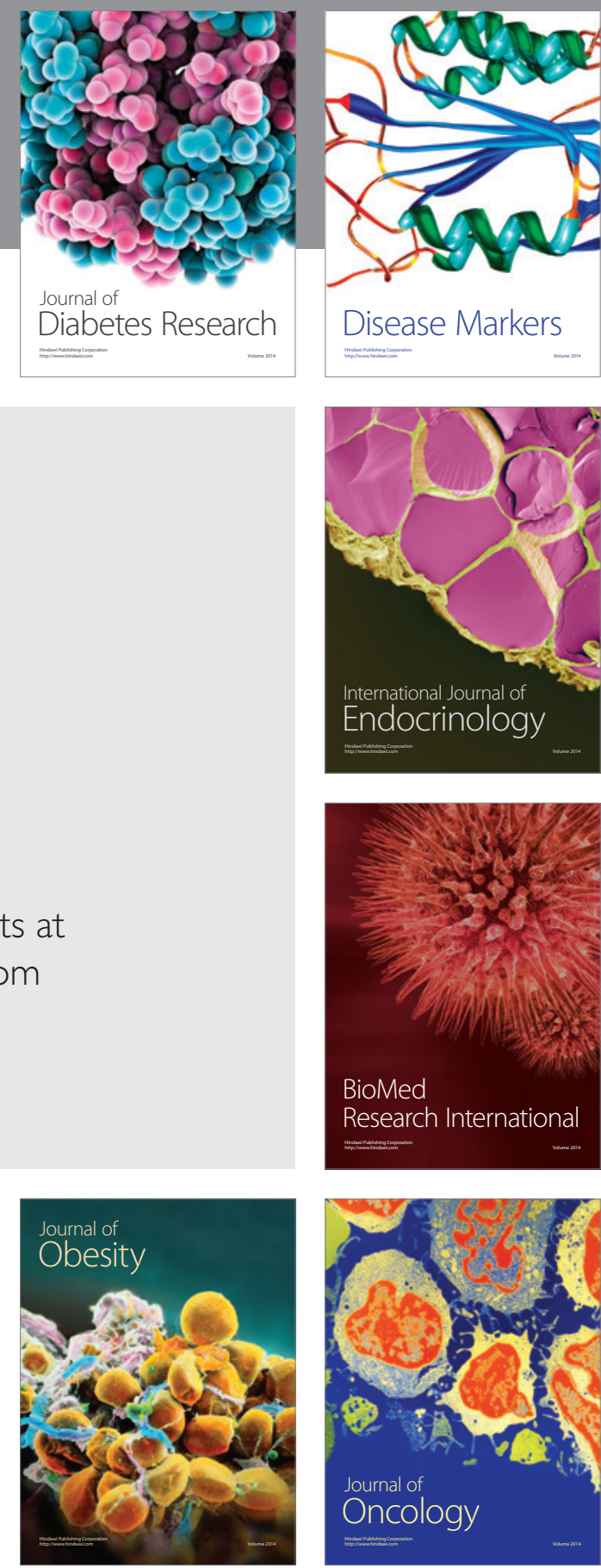

Disease Markers
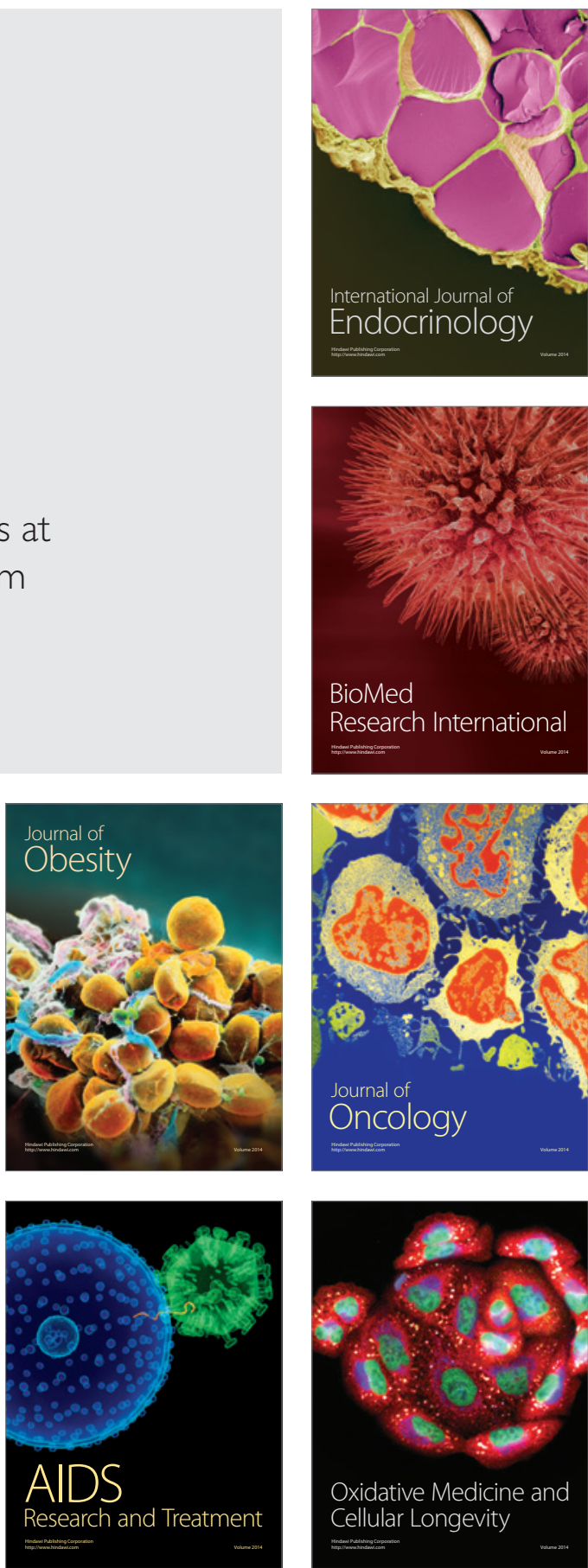